\title{
The Association of Homozygote T Allele of rs2943641 Polymorphism near of Insulin Receptor Substrate 1 Gene in the Susceptibility to Autism
}

\section{Ghaliah Alnefaie*}

Medical Genetic Department, Taif University, Taif, Saudi Arabia

\begin{abstract}
Background: Autism disorder is a neuro-developmental disorder; it is heterogeneous with multiple genes defects that can lead to autism. The incidence has increased from 1980 to $1990,5 / 10,000$ to $37 / 10,000$ respectively. The increase in the frequency has led to huge studies being carried out in this field. The main causes and the pathway of the disease is as yet unclear. However, several reports have been documented that indicate that CNVs and single genes disorders that are involved in multiple pathways have a role in the development of autism. The main genes that are associated with ASD are involved in mTOR/PI3K pathway. MTOR/PI3K pathway is responsible for the growth rate and pruning of cellular-synapse. Therefore, increase the activity of this pathway due to mutations in the upstream or downstream of the pathway it may cause ASD to develop. The aim of this dissertation is to present a new aspect by indicating the association of Homozygote T Allele of rs2943641 Polymorphism in IRS1 that is involved in the PI3K pathway and increase the susceptibility to ASD. The effect of homozygote T allele of rs 2943641 has been previously reported as increasing the expression of IRS1. Increase in the expression leads to an increase in the phosphorylation of PI3K that may hyper-activate the pathway.
\end{abstract}

Methods: An allelic discrimination assay was suggested to determine the most common allelic variation of rs2943641 in autistic patients in Saudi Arabia.

Results and conclusion: If the result indicates an association between the T allele of rs2943641 and ASD, a new aspect in the genetic causes for autism will have been added.

Keywords: Autism; IRS1-Autism; rs2943641 Polymorphism-Autism; IRS-1; rs2943641; T Allele of rs2943641

\section{Introduction and Background}

\section{Introduction}

Most patients who have autistic disorders suffer from some form of developmental delay and severe neuro-developmental disorders have an early onset. Frequently shared common clinical features are deficiency in social interaction, language delay and repetitive behaviour. They can also have a lower IQ than normal with some of them suffering from intellectual disability [1]. The phenotype differs from patient to patient with variable severity. In May 2013 the new Diagnostic and Statistical Manual of Mental Disorders, published the fifth edition (DSMV) and together with the International Classification of Diseases, Tenth Revision (ICD-10) the classification of autism is now dependent on age of impairment in social, language and showing an interest in activities. The most common are autistic disorder is ASD, next is Asperger syndrome AS, and the third is pervasive developmental disorder not otherwise specified PDD-nos [2]. Reviewed by Freitag in 2006, an Austrian-US-American Professor of Child Psychiatry in 1943 was the first to recognise a child with mental retardation who was isolated due to the lack of ability to communicate. Eugen Bleuler named this condition autism using Bleulers schizophrenia criteria which was used to classify the isolated schizophrenia. In the same period 19501960 Hans Asperger recognised a child with similar autistic feature but without mental retardation. Between 1970-1980 Michael Rutter and Lorna Wing investigated the diagnosis and aetiology of autism [3].

\section{Prevalence}

The incidence and the diagnosis of autism has been increasing dramatically raising the concern of parents, physicians and scientists as to the need for more investigations in order to determine if there are any environmental effects that could be causing the elevation in the incidence [4]. In 1980 the frequency was low 5/10,000, however in 1990 in many of the studies carried out in Japan, England, and Sweden to determine the incidence of autism the results show an increase to
$37 / 10,000$ [5]. Recently, US studies indicate that in 8 year olds there is one child in 110 with ASD. This study does not indicate a huge increase in prevalence rather that it is an increase in awareness and the ability of the public and scientists to evaluate and find a specific diagnosis. The ratio of boys to girls is $4: 1$ but, as yet there is no definitive evidence as to the reason for the difference, however interestingly in the severe cases of ASD the ratio becomes 1.8:1 [4].

\section{Clinical feature}

The symptoms of autism most commonly occur in infants before they are three years of age. Parents are usually late in recognising the abnormal features of ASD in their child which could have alerted them; however diagnosis of the condition may also take a considerable time. Therefore, the age of diagnosis is much later than the age of onset, as there are no specific signs or symptoms that can be clearly recognised at an early age. Autism can be diagnosed in adolescents or adults as a result of the symptoms occurring at age when involvement in society is required. The main three symptoms for autism are lack of social involvement, communication and repetitive action [6] (Figure 1).

\section{Inheritance}

It is not yet known what causes autism therefore; twins and family studies are carried out to investigate if the condition is caused by genetic or environmental factors or both. These studies are carried out

*Corresponding author: Ghaliah Alnefaie, Medical Genetic Department, 14 Alsharqiah Street, Taif University, Taif, Saudi Arabia, Tel: +966561216101; E-mail: ghalia70@windowslive.com

Received March 01, 2014; Accepted March 29, 2014; Published April 04, 2014

Citation: Alnefaie G (2014) The Association of Homozygote T Allele of rs2943641 Polymorphism near of Insulin Receptor Substrate $1 \mathrm{Gene}$ in the Susceptibility to Autism. Gene Technology 3: 109. doi: 10.4172/2329-6682.1000109

Copyright: (c) 2014 Alnefaie G. This is an open-access article distributed under the terms of the Creative Commons Attribution License, which permits unrestricted use, distribution, and reproduction in any medium, provided the original author and source are credited. 


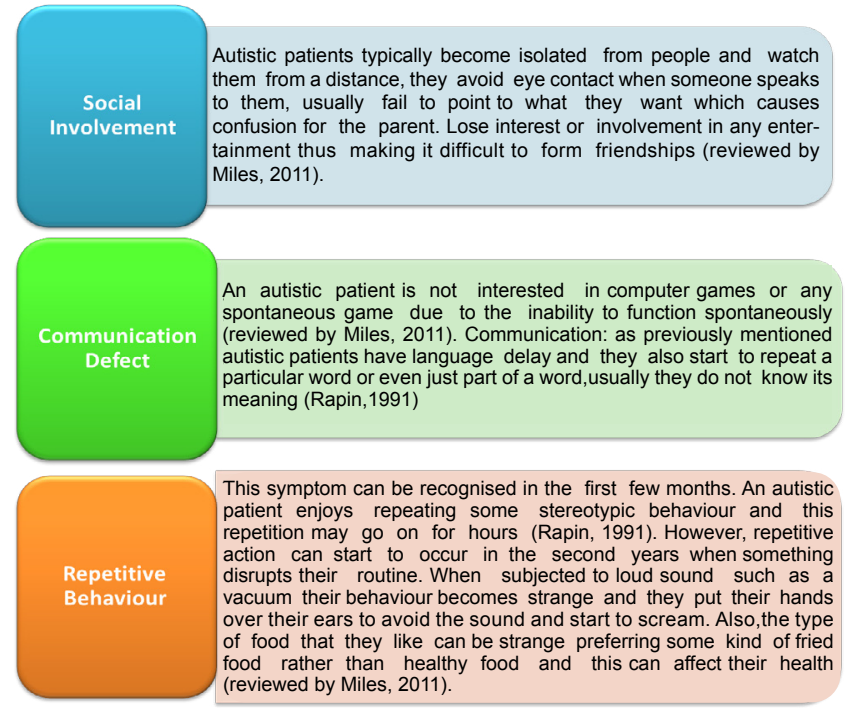

Figure 1: Core autism symptoms $[4,5]$.

in order to illustrate the concordance and recurrence rates. It is also used to determine the pattern of inheritance which has been suggested as being Mendelian [3].

Twins: Freitag in 2006 has been reviewed that, twin studies have determined that, the majority of the causes of autism are genetic. The concordance rate in autistic cases in monozygotic MZ twins is between $36-96 \%$ and $0-30 \%$ in same sex dizygotic DZ twin pairs. This is really surprising as in MZ, they have identical genetic background share $100 \%$ of genetic yet have a different phenotype which suggests that there are modifying factors. The modifying factors are suggested as being environmental as each of these twins has been exposed to different environmental factors [3].

Siblings-ship: Los Angeles-University Utah study in 1989 reported that the recurrence risk for siblings of an autistic child is $8.9 \%$, however if the family has more than one affected child the recurrence risk is $35 \%$. Therefore, the increase of ASD in an autistic family can be determined by comparing the frequency of autistic disorders in relatives of autistic patients with the prevalence of the disease in the population. The prevalence estimated in this study is 9 per 1000 and the recurrence rate in siblings of an autistic family is $10.9 \%$ which is greater than it occurring by chance [3].

Parents: Studies of the parents of autistic children have shown a rate of 10 to $45 \%$ social failure which is due to language difficulties causing problems in communicating and can occur in both fathers and mothers of children with autism [3]. In addition, parents of an autistic child with an unknown genetic factor present with high anxiety disorders involving social phobias, depression, obsessive-compulsive behaviours and it was shown that an increase in the severity of disability in social, communication, cognitive and repetitive behaviour increased the risk of having a child with autism [7].

\section{Clinical diagnosis}

The basic diagnosis for autism is carried out by evaluating the behaviour according to specific guide lines associated with DSM-IVTR. The fifth edition of which has criteria that had not been accepted until now [8].

Physicians can diagnosis an autistic patient by asking particular questions to parents and the patients while also observing specific characteristics in the patient. Autism will be diagnosed in a child of 3 years who has a delay in language development or social communication and abnormality in the pattern to playing [8]. Neurology can help in the diagnosis of autism. For example, in Saudi Arabia they used MRI as a basic technique of diagnosis autism.

Autism can be diagnosed by neurologists using scan images that can illustrate that the brain pattern, Muller et al. in 2001 shows differentiation between autistic patients and normal controls, autism shows abnormality in the function map. The standard magnetic resonances images detect reduction in the pattern of distinct regional activation-deactivation in autism. This result matches the imprinting in the motor function for autism [9].

\section{Environment}

Environmental factors can interact with gene mutations to cause ASD. Exposure to high level of pollution and impairment during gestation such viral infection can also increase the susceptibility of having an autistic child. In addition, testosterone and over activity of the immune system can increase the risk to the foetus of ASD [10].

\section{The history of genetic diagnosis of ASD}

In 1970 the cause of ASD was widely believed to be a biological effect connected to the appearance of aloof behaviour of the mother. However, in 1980 with the increase of the role of genetics in these phenomena and also with the increase in chromosome abnormality which had been noticed in ASD patients many syndromes have been correlated with ASD [5]. In addition, twins and family study linkage analysis have detected a strong correlation between genetic factors and the etiology of ASD that is lacking specific diagnosis criteria [3]. In the late 1990's, whole-genome association studies were carried out to identify specific loci leading to ASD, while the genomic technique such as aCGH was used to identify copy number variation $\mathrm{CNV}$ and also has highlighted a number of interesting loci. Recently, genetic classification of the causes of ASD show, 5\% caused by chromosome abnormality, $20 \%$ caused by CNV and $5 \%$ due to a single gene disorder. 20 to $25 \%$ of the mutated genes in autistic cases were identified and related to ASD, the number identified has been increased due to the use of aCGH. However in $75 \%$ to $80 \%$ of autistic cases the cause is unknown [4].

There are multiple rare familial mutations and environmental factors that can lead to ASD [10]. As well as heterogeneity, there are biological effects that have been hypothesised as being similar to ASD such as having a defect in synaptic function and abnormality in brain function. As a result, whole genome analysis and pathway mechanism studies must be carried out to correlate phenotype-genotype relations [5].

\section{Chromosome rearrangement and CNVs}

Down syndrome: Down syndrome DS is caused by trisomy of chromosome 21 as a result of meiotic non-disjunction or by Robertsonian translocation. Rasmussen et al., in 2001 has indicated in their experiment that, there is a delay in the diagnosis of ASD in Down syndrome patients when compared with the diagnosis with autism in non-DS patient. In this study they suggested that autism should be considered in DS patient, $7 \%$ of DS having ASD. They attempt to diagnose, indicate and fully assess ASD in DS patients as that will facilitate support for them by special education or other supporting elements. They also determined that significant factors lead to the development of ASD in DS patients such as: a history of ASD in a first or second degree relative and early hypothyroidism. 
Copy number variations CNVs and Autism: The expected cause of ASD is CNVs, $5-10 \%$ in non-syndromic, $10-20 \%$ in syndromic patients and schizophrenia 5\% (Table 1) [11]. Reviewed by Bauer and Msall in 2011, Array-CGH is the first choice in diagnosing ASD as it has high resolution, and the ability to detect abnormalities of less than $5 \mathrm{Mb}$ even as small as $1 \mathrm{M}$. Shen, et al., in 2010 determined that aCGH is capable of detecting $18.2 \%$ of CNVs small deletions and duplications in ASD patients. $7 \%$ of the CNVs whereas determined to be abnormal whereas, the remaining $11 \%$ of the CNVs were of unknown significance [12]. Therefore, aCGH is a good technique to use in the detection of de novo small deletions or duplications [13] (Table 1).

Reviewed by Miles, 2011 several CNVs that are de novo or inherited events related to ASD. They found duplications in $15 \mathrm{q} 11.2$ 11.3 in autistic patients which includes the 15q11.2-11.3 region that is documented in OMIM\#608636 as being related to autism. They also reported that, $20 \%$ of deletions in $16 \mathrm{p} 11.2$ are located in the hot spot region that is related to $1 \%$ autism with macrocephaly compared to $60 \%$ of duplications in the same region 16p11.2 that lead to attention deficit hyperactivity disorder ADHD with microcephaly [4]. 16p11.2 is an area involved in the gene that is important in brain structural development [14]. In addition 7q11.23 duplication, Williams syndrome region, has been found to be associated with ASD in $1 \%$ of cases [4].

Single gene disorders: A study has been carried out that has focussed on the analysis of a single gene responsible for ASD in familial, de nova or a spontaneous event and illustrate in idiopathic and syndromic ASD the associate loci and candidate genes, however no specific gene has been identified as being responsible for ASD [10]. They analysed five genes that are considered to be the most likely candidates to produce the ASD phenotype (Table 2). The studies show that, syndromic autism is usually caused by a single gene disorder. Recently, SFARIGENE

\begin{tabular}{|c|c|c|c|}
\hline CNV associated with & High resolution array detects & Inherited & A de novo \\
\hline Non-syndromic & $8 \%$ CNV & $2 \%$ & $6 \%$ \\
\hline Syndromic & $25 \%$ CNV & $18 \%$ & $7 \%$ \\
\hline
\end{tabular}

Table 1: The percentage of the detection rate for autism by high resolution array, and percentage of inherited and de novo event [11]. database has a list of the candidate genes associated with ASD [4].

Rett syndrome: Rett syndromes is caused by a mutation in the $\mathrm{X}$-linked gene MECP2 that codes for methyl CpG-binding protein 2, it has been reviewed by Castro et al., in 2013 that, deficiency in MECP2 leads to alteration in the significant intracellular pathway for example $\mathrm{mTOR} / \mathrm{PI} 3 \mathrm{~K}$ signalling pathway that is related to autism. Approximately $25 \%$ to $40 \%$ of individuals with Rett syndrome are also diagnosed with autism [15].

Genes balance between excitatory inhibitory signals: NRXN1NLGN3\&4-SHANK3 pathway is responsible for maintaining the balance between excitatory and inhibitory therefore; mutation in either of them may cause mental retardation. The association between alteration in the synaptic function and ASD has been observed in 10\% to $30 \%$ of ASD patients who suffer from epilepsy due to an imbalance in excitatory-inhibitory signal [16]. This association has been proved by the detection of mutations in Neuroligins (NLGN) that are postsynaptic cell adhesion molecules. Also, several mutations in SHANK3, NRXN1, CNTNAP2 and CNTNAP3/4, are detected in ASD (Figure 2) [17]. The hypothesis is that the presence of these mutations requires other factors before ASD will be developed and it can also be the cause of other diseases (Table 3).

\section{Genes involved in mTOR/PI3K pathways}

mTOR/PI3K pathway activated by insulin signalling: Phosphoinositide-3kinase mTOR/PI3K pathway that is responsible for the growth rate of cellular-synapse, is one of the most important pathways and includes several genes that are associated with syndromic ASD Bourgeron, 2009 (Figure 3). mTOR/PI3K pathway connects the extra cellular insulin signalling to activate mTOR. The signalling links to $\mathrm{PI} 3 \mathrm{~K}$ involve insulin receptor substrate IRS. This will activate the PI3K/ mTOR pathway which is responsible for pruning and the formation of the synapse. Abnormality in the shape and the size of neuron increases the susceptibility for autism. Therefore, mutation in the upstream may lead to an increase in the activity of this pathway that leads to ASD [16].

TSC1/TSC2, and PTEN work in one pathway like a negative control

\begin{tabular}{|l|l|}
\hline Location & Gene Name \\
\hline $\mathbf{7 q 3 6 . 2}$ & EN2 Engrailed homolog 2 gene \\
\hline $\mathbf{1 5 q 1 1 q 1 3}$ & GABR Gamma amino butyric acid receptor genes \\
\hline $\mathbf{1 6 p 1 1 . 2}$ & OXTR oxytocin receptor genes \\
\hline $\mathbf{7 q 2 1 - q 3 6}$ & RELN Reelin gene \\
\hline $\mathbf{1 7 q 1 1 . 1 - q 1 2}$ & SLC6A4 Serotonin transporter gene \\
\hline
\end{tabular}

Function Involved in cerebellum development and it has essential role during development in segmentation

Regulate brain cell migration, differentiation, and synapse formation Involved in the response to stress and in social skills such as empathy Involved in neuronal migration in the developing brain Could account for phenotypic expression of happiness

Table 2: Five genes associated with autism [10].

\begin{tabular}{|c|c|c|c|c|}
\hline 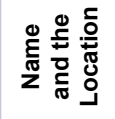 & $\begin{array}{l}\text { Neurexin1 NRXN1 } \\
\text { 2p16.3 }\end{array}$ & $\begin{array}{l}\text { Contactin-associated } \\
\text { protein-2 CNTNAP2 } \\
7 \text { q35 }\end{array}$ & $\begin{array}{l}\text { Mltiple ankyrin repeat } \\
\text { domains } 3 \text { SHANK3 } \\
22 q 13\end{array}$ & $\begin{array}{l}\text { Neuroligins } 3 \& 4 \text { NLGN } 3 q 13.1 \\
\text { and NLGN } 4 \text { Xp22.3 }\end{array}$ \\
\hline 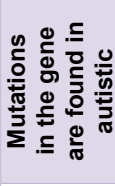 & $\begin{array}{l}\text { Wiśniowiecka-Kowalnik, et al., in } 2010 \text { indicated } \\
\text { that } \sim 380 \text { kb deletion in women suffering from } \\
\text { Asperger syndrome, had anxiety and depression } \\
\text { they also found the same deletion in four of her } \\
\text { children who were affected by ASD but they } \\
\text { didn't find the deletion in an unaffected child }\end{array}$ & $\begin{array}{l}\text { Tan et al., in } 2010 \text { investigating } \\
\text { an autistic patient using MRI and } \\
\text { they found that homozygotes } \\
\text { rs } 7794745 \mathrm{~T} \text { allele is the risk allele } \\
\text { may has association with autism }\end{array}$ & $\begin{array}{l}\text { Durand et al., in } 2007 \\
\text { detected proband autistic } \\
\text { patient carried a de novo } \\
\text { deletion on } 22 \text { q13 the } \\
\text { breakpoint SHANK3 was } \\
\text { located in intron } 8\end{array}$ & $\begin{array}{l}\text { Laumonnie et al., in } 2004 \text { investigated a } \\
\text { French family } 12 \text { members with mental } \\
\text { retardation caused by deletion on Xp22.3 } \\
\text { NLGN4. } \\
\text { Reviewed by Stamou et al., in } 2012 \text { point } \\
\text { mutation R451C in NLGN3 }\end{array}$ \\
\hline 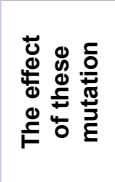 & $\begin{array}{l}\text { Mutations in NRXN1 do not increase the number } \\
\text { of synaptic sites. However, they increase the } \\
\text { strength of existing synaptic and usually it } \\
\text { presents the repetitive behaviour in ASD [29] }\end{array}$ & $\begin{array}{l}\text { Allelic variation of CNTNAP2 a } \\
\text { member of the neurexin family } \\
\text { is linked with change in the white } \\
\text { and grey matter in the frontal lobar } \\
\text { region [26] }\end{array}$ & $\begin{array}{l}\text { Mutations or small } \\
\text { rearrangements in SHANK3 } \\
\text { usually lead to impairment in } \\
\text { language development and } \\
\text { cognitive disorder phenotype } \\
\text { in autistic patient }\end{array}$ & $\begin{array}{l}\text { Mutations in NLGN3 and NLGN4 are } \\
\text { associated with many psychiatric disorders, } \\
\text { social imprinting with language disabilities }\end{array}$ \\
\hline
\end{tabular}

Table 3: Genes regulate the excitatory inhibitory signals [26-32] 


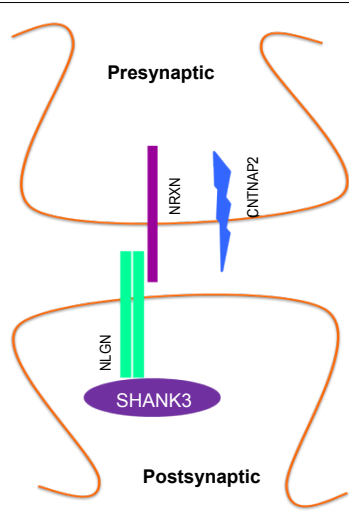

Figure 2: NLGN3\&4-NRXN1-SHANK3-CNTNAP2 are important in the stability of synaptic function and also has a role in social involvement. NLGN3\&4: are post synaptic proteins that bind with SHANK3, Neuroligin-neurexins regulate synaptic plasticity by control of the generation of excitatory and inhibitory synapses in vitro [16]. NRXN1: is presynaptic in that it binds with NLGN. Binding of NLGN-NRXN is mediated synaptic development. Therefore, it promotes the synaptic formation. SHANK3: is postsynaptic protein, connected the receptors in postsynaptic membrane to the cytoskeleton and have a role in the signalling pathway that regulating the shape and size of dendritic synaptic NLGN. CNTNAP2: presynaptic protein, it is highly restricted to frontal and interior temporal lobes striatum and dorsal thalamus [26]

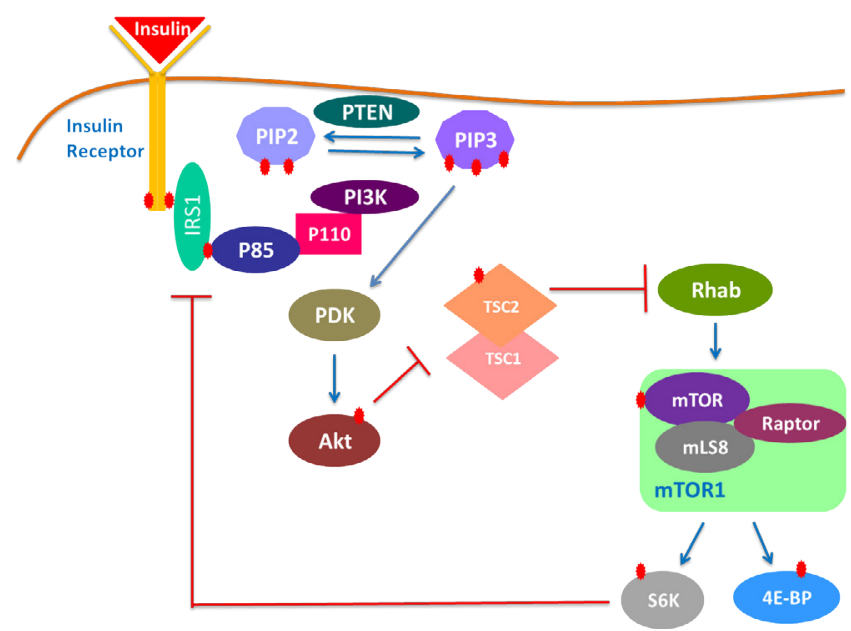

Figure 3: Insulin signalling activate mTOR/PI3K pathway. Insulin and insulin like growth factor corroborating with insulin receptor that will phosphorylate insulin receptor substrate IRS1. In turn IRS1 corporate with p85 and p110 to activate the PI3K. Once the PI3K has phosphorylated, the 4,5-phosphatidylinositolbiphosphate (PIP2) produces phosphatidylinositol 3,4,5-triphosphate (PIP3), which plays a role in the activation of PDK. PDK in turn activates the pathway in phosphorylating Akt that plays a critical role in inhibiting TSC1/TSC2 which inhibits Rheb. By this mechanism Rheb is now active and can activate mTOR1. MTOR1 which will activate the $4 \mathrm{E}$ BP1 and S6K. Negative regulation of the pathway is by PTEN and TSC1/ TSC2. PETN negatively regulates the 4,5-biphosphate (PIP2) and produces phosphatidylinositol 3,4,5-triphosphate (PIP3) while, TSC1/TSC2 regulates the Rheb mechanism. In addition the negative regulator for this pathway is also mediated by S6K which can block the insulin signalling by inhibiting the IRS [16].

for mTOR in the insulin signalling pathway that is associated with ASD and is responsible for cell growth and development which leads to social imprinting [10].

Phosphatase and tensin homolog (PTEN): PTEN is a tumor suppressor protein that is a regulator of cell proliferation and differentiation, PTEN encodes a ubiquitously expressed and important protein which has a lipid phosphatase function. PTEN acts as an inhibitor in PI3K the the pathway by removing phosphate from the signalling molecule phosphoinositide-3,4,5-triphosphate PIP3. PTEN mutation was reported by Herman et al., in 2007 to be in autistic families. Sequencing the PTEN was carried out in two families that had Cowden syndrome occurring in the parent Cowden syndrome presents where there are mutations in PTEN and they found a nonsense mutation R130X within exon 5 PTEN is present in the essential phosphatase domain of the protein and Y178X in exon 6 in an autistic patient with macrocephaly measuring $>2.5$ standard deviations SD above the mean. Therefore, sequencing PTEN for autistic patents that have macrocephaly is recommended [18].

Tuberous sclerosis 1 and 2 (TSC1 and TSC2): Tuberous sclerosis is a multisystem autosomal-dominant disorder where the loss of function occurs as a result of mutation in either of TSC1 genes in $9 \mathrm{q} 34$ or the TSC2 gene in 16p13.3. The gene product of TSC1 is known as hamartin, and the product of TSC2 is tuberin. Approximately $10 \%$ to $30 \%$ of cases of tuberous sclerosis are due to mutations in TSC1, $90 \%$ to $70 \%$ due to mutations in TSC2. Approximately $30 \%-60 \%$ of patients with tuberous sclerosis complex TSC have autism [16].

TSC acts as a negative regulator for the PI3K pathway by regulating the upstream of mTOR and downstream of Akt. TSC2 inhibits the activation of $\mathrm{S} 6 \mathrm{~K}$ and $4 \mathrm{E}-\mathrm{BP} 1$ by blocking the Akt activity [19]. Chiang et al., in 2013 determined that several missense variations in TSC1 and TSC2 in probands and where inherited increase the susceptibility to autism.

\section{The rs2943641 polymorphism of the IRS1}

\section{IRS1 Structure and Function}

Sun et al., in 1991 detected that, insulin receptor substrate 1 IRS1 encoding protein which phosphorylated by insulin receptor kinase is involved in the mTOR/PI3K pathway which is in association with ASD. IRS1 is located on the long arm of chromosome $2 \mathrm{q} 36.3$ has responsibility for insulin resistance and is associated with type II diabetes "non-insulin-dependent diabetes mellitus" NIDDM.

Interestingly IRS 1 is not included in crucial enzymatic activity it and acts as activation for different pathways after phosphorylation with tyrosine by insulin receptor kinase. Normally, IRS1 protein is phosphorylated when it interacts with insulin or insulin like growth factor by insulin receptor tyrosine kinase to regulate the insulin signalling process. IRS1 bind usually interacts with protein included in the SH domain e.g. PI3K, p85 and Grb2. Therefore after, the insulin signal has phosphorylated the insulin receptor kinase, IRS1 will then phosphorylate and corroborate with p85 and p110 to activate PI3K. IRS1 therefore plays a critical role in the activation of the downstream of PI3K pathway [20].

\section{IRS1 domains structure and function}

Insulin receptor substrate1 IRS1 consists of multiple domains for structure and functional acts. Normally under insulin stimulation $632 \mathrm{Y}$ motif in IRS1 binds with insulin receptor kinase to activate IRS1 that activate the PI3K pathway. However, in a case of hyperinsulinism the increase the insulin signalling for activating the PI3K pathway is inhibited by phosphorylation of IRS1 at Ser-636/639 through mTOR1 [21] (Table 4).

Tzatsos in 2009 found that, SAIN domain is a critical domain in IRS1 having a role in regulating the phosphorylation of IRS1 at Ser$636 / 639$ by mTOR1 (Figure 4). Raptor plays an important role through 


\begin{tabular}{|l|l|}
\hline Serine types & Effect \\
\hline Ser312 & $\begin{array}{l}\text { Inhibit the insulin signalling via block the IRS1 interact with insulin } \\
\text { receptor kinase. }\end{array}$ \\
\hline Ser270 & $\begin{array}{l}\text { Motif around serine 270 can enhance the interaction between IRS1 } \\
\text { and insulin receptor kinase and it found in the phosphotyrosine- } \\
\text { binding (PTB) domain in IRS1. }\end{array}$ \\
\hline Ser 629 & $\begin{array}{l}\text { When increasing the phosphorylation of ser629 the } \\
\text { phosphorylation of ser636 will reduce and acts as an enhancer } \\
\text { for insulin signalling in PI3K pathway [21] }\end{array}$ \\
\hline Ser307 & $\begin{array}{l}\text { They are near the 632Y motif and their function is to reduce the } \\
\text { insulin stimulation of the PI3K pathway via the IRS1, through }\end{array}$ \\
\hline Ser1101 & $\begin{array}{l}\text { negative feedback it sent from Raptor which interacts with mTOR } \\
\text { and mLS8 to block the IRS1. }\end{array}$ \\
\hline Ser636 & \\
\hline Ser639 &
\end{tabular}

Table 4: The mechanism of serine phosphorylation of IRS1 [20,21].

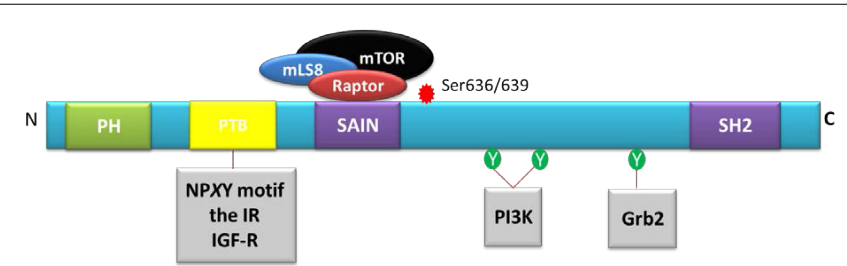

Figure 4: The insulin receptor substrate 1 IRS1 domains. $\mathrm{PH}$ domain pleckstrin homology domain located in the $\mathrm{N}$ terminal that assists in presenting the IRS1 proteins in close proximity to the insulin receptor kinase. PTB domain: flanked the PH domain and acts as binding to the NPXY motif of the insulin receptor IR and other receptors such as insulin-like growth factor-1 IGF-R1. SAIN domain: Shc and IRS-1 NPXY binding domain receive the inhibitor signal through the mTOR-mLS8-Raptor complex which can phosphorylation ser636/639 that inhibits the IRS1 downstream signaling. The carboxyl terminal involved contains multiple Tyr phosphorylation e.g. PI3K, Grb2. That also acts as docking sites for $\mathrm{SH} 2$ domain [22].

interaction with mTOR1 and mLST8 to form mTOR1 complex that regulates the phosphorylation of IRS1 at Ser-636/639 and has been found in noninsulin-dependent diabetes mellitus the activity of Akt is reduced with the increase in phosphorylation of IRS1 at Ser-636/639 [22].

\section{The effect of rs2943641 in IRS1}

rs2943641 single nucleotide polymorphism SNP located in Inter-genic region $500 \mathrm{~kb}$ upstream from the IRS1 in chromosome 2 q36 is likely to have an effect on expression. The HGVS named is g.227093745T $>$ C. Meaning that the ancestral nucleotide is a $\mathrm{T}$ at position 227093745 in the genomic DNA region has been changed to a C. the minor allele is ' $\mathrm{T}$ " (Figure 5) [23].

\section{Explain the rs2943641 hypothesis}

Autism is a neuro-developmental disorder with an early onset; autistic patients are unique with due to their repetitive behaviour. The genetic cause is still complicated as we know it is heterogeneous with multiple effects that can lead to ASD. In some case the individual has inherited a genetic defect that when interacting with environmental factors can present a serious disease such as autism. Recently, several studies have been carried out and these revealed essential information for example there are studies that illustrate many pathways that interact with autism. Several syndromes have been also reported are associated with autism as mention before [4]. This research has been carried out to add a new aspect for the genetic causes that can elevate the susceptibility to autism.

rs2943641 polymorphism has been chosen to be the main hypothesis of this study. This polymorphism is located near of IRSI which is a critical element in the activation the downstream of this in the mTOR/PI3K pathway as a response to the stimulation insulin signalling as mention before [20].

Rung et al., in 2009 has investigated that, the effect of the rs2943641 in transferring insulin signaling that stimulates PI3K pathway. They found that the C allele of rs 2943641 has reduced the IRS1 expression that is associated with a decrease in the phosphorylation of PI3K under insulin signalling stimulates. The reason for this is that the $\mathrm{C}$ allele reduces the insulin sensitivity leading to insulin resistance. Therefore, this foundation has provided the hypothesis of rs2943641 variation has a role in the IRS1 expression that affects the activity of the PI3K pathway.

The hypothesis is that, this research has determined that specific variation of polymorphisms can affect over-expression of IRS1 gene. This suggested that an increase in the expression of IRS 1 can increase the activity of the PI3K pathway that may lead to increase the susceptibility for ASD [16]. Maglio et al., in 2013 determined that homozygote T allele of rs2943641 results in lowering the insulin resistance in other word increase the insulin sensitivity through over-expression of IRS1.

This hypothesis dose not suspect that the rs2943641 is the main cause of autism but rs2943641 may one of the genetic factors that triggers the susceptibility for autism through increasing the activity of the PI3K pathway under the insulin signalling. On the other hand, rs2943641 may not increase the susceptibility for autism however, if it may links with another alteration it could then have role in causing autism. No studies have determined that this association therefore, this study aims is to determine the association of Homozygote T Allele of rs2943641 Polymorphism near of IRS1 and increase the susceptibility to ASD.

\section{Method "Designing the Experiment"}

\section{Aim of the investigation}

The aim of investigation is to determine the association of Homozygote T Allele of rs2943641 Polymorphism in IRS1 and increase the susceptibility to ASD.

\section{Facilities}

This experiment can be carried out in a laboratory that has polymerase chain reaction PCR availability, and 7700 sequence detection system SDS to measure the florescent intensity after the TaqMan assay proses.

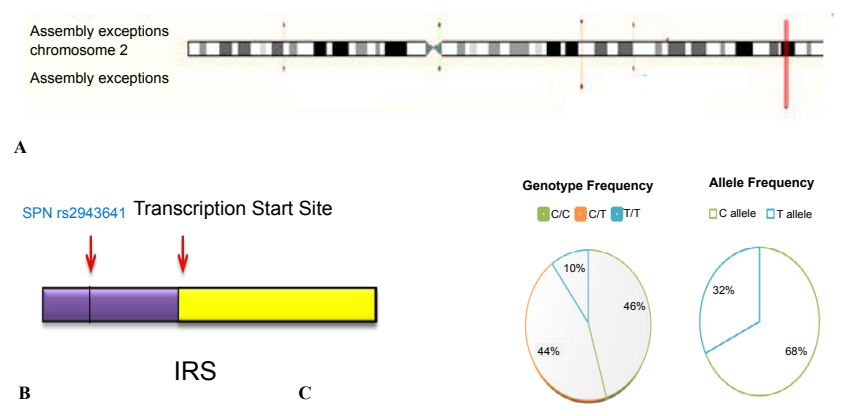

Figure 5: location of the rs2943641 in the IRS1: A: The Ensembl website presents the location of the SNP in red box in chromosome 2: 227,093,695$227,093,795$ [23]. B: rs2943641 is located upstream from the transcript start site of the IRS1. C: the distribution of $\mathrm{C}$ allele is almost two thirds of the distribution of T allele. CC allele is the most common genotype with $46 \%$ then the CT allele with $44 \%$, while TT allele accounts just $10 \%$ [27]. 
Citation: Alnefaie G (2014) The Association of Homozygote T Allele of rs2943641 Polymorphism near of Insulin Receptor Substrate 1 Gene in the Susceptibility to Autism. Gene Technology 3: 109. doi: 10.4172/2329-6682.1000109

\section{Strategy}

Strategy plan has been determined to obtain the desired result. The strategy is clearly explained in Figure 6.

\section{Design the allelic discrimination assay}

To design allelic discrimination assay that is able to differentiate between the individual who is homozygote for the T allele of rs 2943641

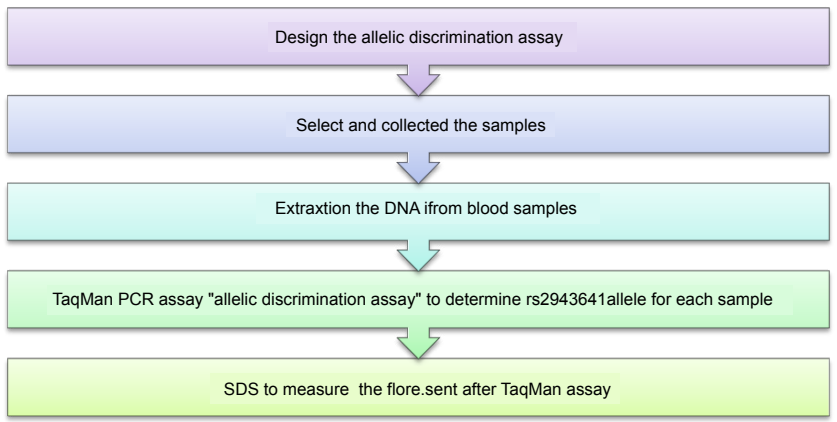

Figure 6: Strategy of the project.
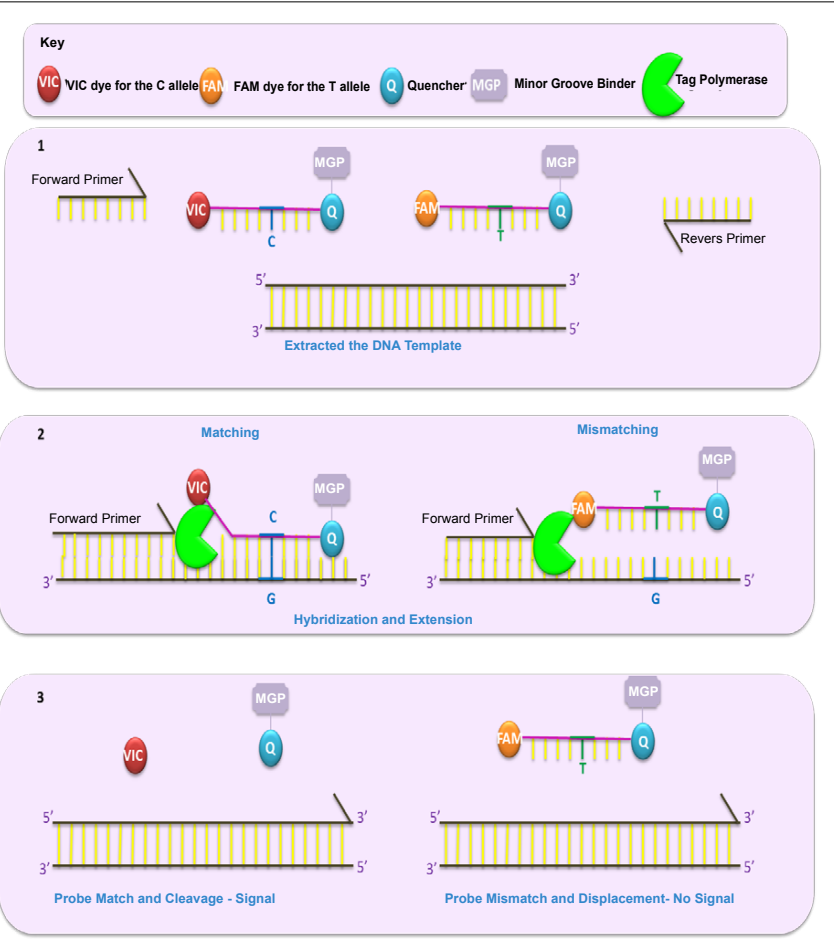

Figure 7: TaqMan assay is used to distinguish between the $C$ and T allele in rs2943641. The DNA was extracted to carry out the experiment, panel 1 presents the component of the assay: forward and reverse primers for the flanking sequence of the SNP. Two probes were used with two different reported dyes, VIC dye labelled the probe which was used to detect the $C$ allele and FAM dye labelled the probe which was used to detect the T allele. Panel 2 presents the hybridizing and the extension: after denaturation the template, the probes and the primers anneal in the single strand template. The probes have annealed before the primer due to the melting temperature for the probes being higher by $10^{\circ} \mathrm{C}$ than the melting temperature of the primers. The $5^{\prime}-3$ exonuclease activity of Taq polymerase degrades the matching probes which hybridize to the template. Degradation leads the florescent being released from the probes. Panel 3 shows the dye emission and produce signalling after the cleavage by exonuclease activity of the Taq polymerase in the matching probe. However, in the mismatch the probe will be displaced without producing signalling due to no cleavage occurring [24].

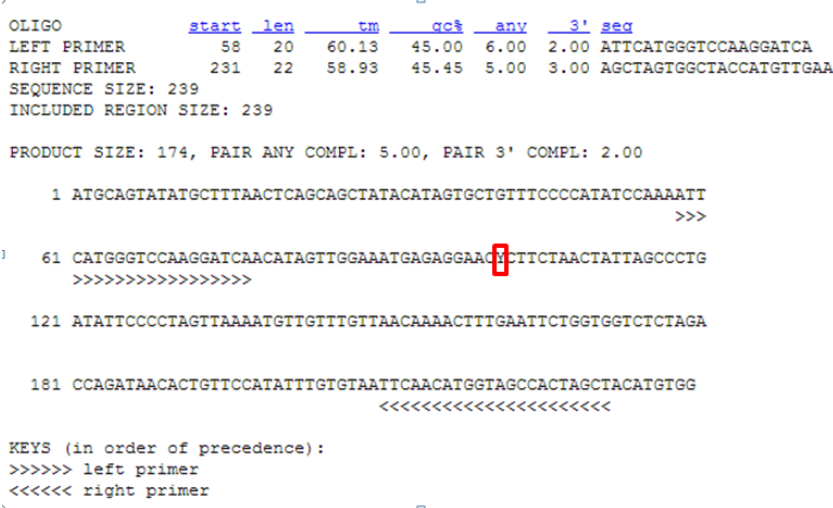

Figure 8: Data about the forward and reverse primer for the flanking sequence of rs2943641: The primers were designed by Primer3 web. The polymorphism presented by the red box in the flanking sequence and the forward primer symbol is $\gg>$, while the reverse primer symbol is $\ll<<[3]$.

\begin{tabular}{|l|l|l|}
\hline Probe & C allele probe & T allele probe \\
\hline Sequence & GAAATGAGAGGAACCCTTCTAACTA & AGGAACTCTTCTAACTATTAGCCC \\
\hline Length & $25 \mathrm{bp}$ & $24 \mathrm{bp}$ \\
\hline TM & $67.45^{\circ} \mathrm{C}$ & $66.98^{\circ} \mathrm{C}$ \\
\hline
\end{tabular}

Table 5: The $\mathrm{C}$ and $\mathrm{T}$ allele probes design for TaqMan

The polymorphism highlighted by yellow.

polymorphism from the individual who is homozygote for the $\mathrm{C}$ allele and who is heterozygote. TaqMan PCR assay was chosen as the allelic discrimination assay, TaqMan is $5^{\prime}$ nuclease assay used in genotyping studies to distinguish allelic variations of rs2943641. This technique is based on the $5^{\prime}-3^{\prime}$ exonuclease activity of the Taq polymerase to cleave the labelled probes which are hybridized to the complementary sequence that illustrates fluorescent signals during PCR (Figure 7) [24].

For the rs2943641 SNP TaqMan required two probes one containing the $\mathrm{C}$ allele and the other for the $\mathrm{T}$ allele. Each probe is labelled with 5 ' reported dye that has different colours to distinguish between the $\mathrm{C}$ allele probe and the $\mathrm{T}$ allele probe, and a 3' quencher dye that blocks the reported dye from releasing the fluorescence (Figure 7) [24].

In the TaqMan PCR probes are designed to anneal to the specific SNP site sequence. The flanking sequence has been designed using the Ensembl website (Figure 8). The sequence is amplified by specific unlabelled primer sites which are designed by Primer3 left primer ATTCATGGGTCCAAGGATCA and right primer AGCTAGTGGCTACCATGTTGAA the melting temperature TM approximately $59.53^{\circ} \mathrm{C}$ and the amplicon length is $174 \mathrm{bp}$ (Table 5). The two probes of the TaqMan assay will be designed, one for the $\mathrm{C}$ allele labelled with VIC dye as fluorophores and MGB as Quenchers and the other probe for the $\mathrm{T}$ allele labelled with FAM dye as Fluorophores and MGB as Quenchers the probes then sequencing (Figure 9).

Life technologies are a large company that can provide several brands of laboratory products. This experiment has suggested that the primers and the probes should be ordered from life technology as that will facilitate ordering the single tube custom TaqMan SNP genotyping assays kit. The kit contains two primers and two different probes. TaqMan master mix is ordered separately. Real time PCR instrument will automatically run and analysis the assay. Single tube custom TaqMan SNP genotyping assays kit can run 1,500 reactions [24].

\section{Sample source}

Blood sample can be sourced from Prince Salman Central for 


\begin{tabular}{|c|c|}
\hline TaqMan Probes & Primers \\
\hline $\begin{array}{l}\text { - Length of the probe is between } 20-30 \\
\text { bases } \\
\text {-The percentage of the CG in the } \\
\text { product should be between } 20 \%-40 \% \\
\text { - Melting temperature TM of the prods } \\
\text { is } 68-70^{\circ} \mathrm{C} \text { high by } 10^{\circ} \mathrm{C} \text { than the TM } \\
\text { of the primer } \\
\text { - Repeated nucleotides in the } \\
\text { sequence should be avoided } \\
\text { - The polymorphism Tor C should be in } \\
\text { the central of the probe } \\
\text { - In the C allele probe the reported dye } \\
\text { is VIC at the } 5^{\prime} \text { end } \\
\text {-In the T allele probe the reported dye } \\
\text { is FAM in the } 5^{\prime} \text { 'end } \\
\text {-The quencher dye is MGP in the } 3^{\prime} \\
\text { end for the both probes }\end{array}$ & $\begin{array}{l}\text { - The length of the primer should be } \\
\text { between } 20-24 \text { bases } \\
\text { - The percentage of the CG in the } \\
\text { product should be between } 20 \%-60 \% \\
\text {-Melting temperature Tm of the prods } \\
\text { is } 58-60 \text { oC } \\
\text { - Repeated nucleotides in the sequence } \\
\text { should be avoided } \\
\text { - Self complimentary should be a voided } \\
\text { - Reverse and forward primer should } \\
\text { not be complementary to each other } \\
\text { - The reverse and forward primer should } \\
\text { be close to the probes with out } \\
\text { overlapping } \\
\text {-The } 3 \text { ' end of each primer should } \\
\text { match the template } \\
\text {-The amplicon size no more that } 200 \text { bp }\end{array}$ \\
\hline
\end{tabular}

Figure 9: The design criteria for TaqMan probes and the primers (Life technology).

Disability Research in Saudi Arabia; centre has a blood bank for different types of disability patients that include autistic. patients The blood sample can be provided by the following strategy begin by filling a form explaining to which purpose this sample will be used and if it is for scientific research, if it is ethically acceptable and must follow legal criteria to obtain the approval from the Ministry of Health with this approved the centre will give the sample for free. Normal control samples can be obtained from the blood bank centre in Saudi Arabia which has the blood of the normal children coming from screening tests. These should follow the same criteria. To obtain a good result in these studies 300 samples from autistic patients can be used plus 100 samples from normal individuals used as a control.

\section{Procedure of the assay}

DNA extraction: After collection of the sample, extraction of the DNA from the blood is the critical step in DNA analysis. Therefore, the quality and the accuracy of the result depend on the isolation process. Furthermore, the method used to isolate the DNA depends on the sample type and the storage factor. In this experiment blood samples are collected and stored in a perfect way. DNAzol BD reagent kit from Life technology was ordered to extract 10-20 $\mu$ g DNA from $500 \mu \mathrm{L}$ bloods [24].

Prepare the control samples: Run the samples using the TaqMan assay and select the first three samples which represent a homozygote of $\mathrm{T}$ allele, a heterozygote $\mathrm{CT}$ allele and a homozygote of $\mathrm{C}$ allele then sequencing them to confirm the result. If the results from sequencing have confirmed the results from the TaqMan assay these three samples can be used as positive controls. If the results are not confirmed that means the polymorphism and the amplification are not in the right location therefore the primers and the probes will require being redesigned. Negative controls also should be used to insure the accuracy of the assay this is done by running all TaqMan assay components with the DNA replaced by water.

TaqMan assay procedure: After the DNA was extracted the TaqMan assay was carried out using the negative and positive controls and run in each reaction if it is possible. TaqMan can distinguish between the homozygote $\mathrm{C}$ allele, the homozygote $\mathrm{T}$ allele and the heterozygote $\mathrm{TC}$ allele that will be illustrated by measuring the fluorescence which are emitted on the reported day.

There is useful software which facilitates analysis of the data e.g. Sequence detection systems SDS that records the fluorescent signal for the two reported dyes and are reported by SDS. That will present the data of rs2943641 in a plot or a graph by present homozygote $\mathrm{C}$ allele, heterozygote CT allele and homozygote C allele (Figure 10) [24].

Estimated the cost of the experiment: The estimated cost for the 400 samples can only be roughly calculated due to the possibility that potential problems could lead to re-runs some samples. Therefore, extra cost may need to be added (Table 6) [24].

\section{Results and Conclusion}

The incidence of autism has increased in the last ten years dramatically; however the main genetic reason has been unclear until now. The guilty feelings of parents of autistic patient have risen and they want to know where the defect comes from, most of the cases are de novo or spontaneous mutation, however it has been documented that, if the family already has an autistic patient the possibility that the next baby will suffer from ASD is increased as mentioned previously. It is commonly agreed that autism is heterogeneous and multiple gene defects can cause it. Recently, the studies revealed that many CNVs are related to autism. Several genes involving different pathways have been reported as being associated with ASD. Moreover, autistic symptoms have been present in different syndromes. At present there is no cure for ASD due to the causes being as yet unclear, therefore clinical care attempts to develop the quality of the life for autistics by involving them in special courses that trains them how they can develop language ability, behaviour and increase their confidence to become involved in a social environment.

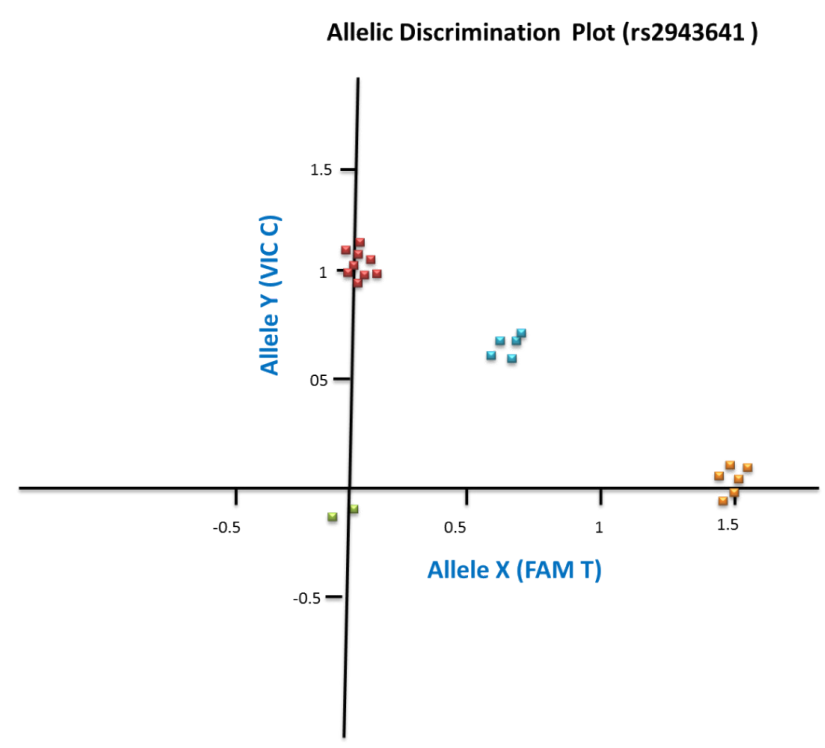

Figure 10: SDS Plot shows the result of TaqMan assay for rs2943641 after the PCR amplification SDS present the data of the polymorphism. The homozygote $C$ alleles are represented by the red color according to the VIC dye, homozygote $\mathrm{T}$ alleles are represented by the orange color according to the FAM dye and TC heterozygote alleles with blue color is a mix between the two dyes. Negative control is represented by the green color [24].

\begin{tabular}{|l|c|c|}
\hline Elements & Enough for how many samples & Cost \\
\hline DNAzol BD reagent kit X4 & 100 sample X4=400 samples & $190 £ \times 4=760 £$ \\
\hline Master Mix & 30,000 samples & $300 £$ \\
\hline $\begin{array}{l}\text { The single tube custom } \\
\text { TaqMan SNP genotyping } \\
\text { assays kit }\end{array}$ & 1,500 reaction & $225 £$ \\
\hline Total & & $1,285 £$ \\
\hline
\end{tabular}

Table 6: Estimated cost for the experiment, the 400 samples for free but required to take the approved. 


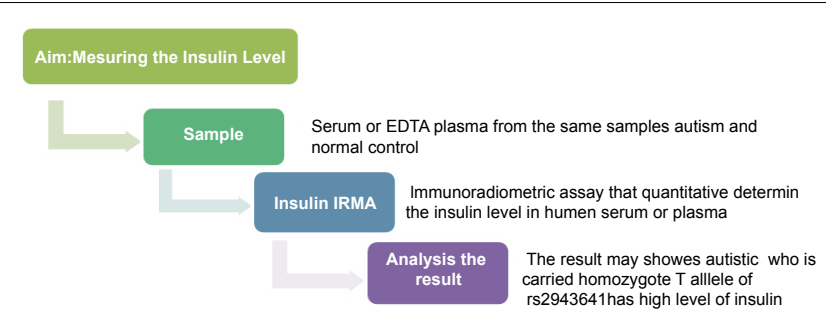

Figure 11: Further experiment can be done as a next step to investigate the role of rs2943641 in autistic patient. The aim and the method are proposed in the figure.

This study tends to concern focusing in new areas to added more information in a new direction. This has been done by investigating the association of Homozygote T Allele of rs2943641 Polymorphism in increasing the susceptibility for autism. The rs 2943641 is located near of IRS1 which is involved in the mTOR/PI3K pathway which is known to be responsible for the growth rate of cellular-synapse; IRS1 can phosphorylate PI3K under the insulin signalling. Increase in phosphorylation can increase the activity of the pathway which has been identified as being associate with autism due to it begin responsible for pruning and the formation of the synapse. Therefore, alteration in the expression of IRS1 can change the activity of mOTR/ PI3K pathway increasing the expression of IRS1 that may up-regulate the pathway, while a decrease in the expression may down-regulate the pathway. Homozygote T allele for rs2943641 has been reported as affecting an increase in the expression of IRS1 leading to low insulin resistance in other word increase the insulin sensitivity. The aim of this study is to identify the association of the homozygous $\mathrm{T}$ allele of rs2943641 polymorphism in autistic patient and comparing them with normal.

Since IRS1 can play an essential role in the activation of the mTOR/ PI3K pathway through phosphorylated PI3K, the polymorphism near of IRS1 role in the susceptibility to autism appear to be sensible? If the result of the experiment shows agreement with the hypothesis i.e. the frequency of the homozygote T allele of rs 2943641 is more in autistic patients than the normal control, while the homozygote $\mathrm{C}$ allele for the polymorphism is low. This would indicate that, rs2943641 is associated with autism due to the effect of homozygote $\mathrm{T}$ allele which has been reported as leading to low insulin resistance with over-expressed IRS1 that in time will increase the phosphorylation of PI3K which may cause hyper-activity of the pathway under the insulin signalling stimulate. Of course, the result may show disagreement with hypothesis, the frequency of homozygote $\mathrm{T}$ allele of $\mathrm{rs} 2943641$ in autistic patients being lower than the normal control, while the frequency of $\mathrm{C}$ allele for rs2943641 higher in autistic patient that the normal control. Therefore, rs2943641 will indicate not have a direct association with autism. However, it may alter the function of other causes of autism. The $\mathrm{C}$ allele has been documented as being associated with low expression of IRS1 leading to high insulin resistance that may alter another causative for autism.

Stern in 2011 has attempt in her hypothesis to connect and explain the relation between the increase in the incidence of autism and hyperinsulinism. Therefore, according to this hypothesis if the result agree with hypothesis further investigation can been carried out by measuring the insulin level in the same samples in autistic and normal control (Figure 11). If the insulin level is indicted as being high in autistics patients who carry the $\mathrm{T}$ allele of the polymorphism then that can confirm the hypothesis that hyperinsulinmis can increase the insulin signalling that hyper-activate the mTOR/PI3K pathway in autistic who are carried the $\mathrm{T}$ allele due to they have high insulin sensitivity. On her other hand, the result may show no alteration in the insulin level in autistic patients but unless hyperinsulinmis hypothesis can be explain be other alternative.

This study was aiming to find a new aspect of polymorphism in IRS1 having a association in the increase in the susceptibility for autism and as IRS1 is in the PI3K pathway therefore, this research will also confirm that the pathway has an essential role in autism [32-36].

\section{Acknowledgements}

I would like to thank Dr. Maria Jackson for her help during the year and her support in giving useful comments and advice on this dissertation.

I would like to thank Dr. Maria Jackson, Dr. Leah Mark and Dr. Edward Tobias for the great knowledge that they provided many thanks.

I would like to thank the Ministry of Higher Education in Saudi Arabia for their financial support and their care in helping me to reach this point.

I would to thank my parents for their love and support while I was taking this step to increase my knowledge and my sister and also brothers for their companionship, which facilitated life in a foreign country.

I would like to thank my friends for their support in the good and bad times.

\section{References}

1. Carter MT, Scherer SW (2013) Autism spectrum disorder in the genetics clinic: a review. Clin Genet 83: 399-407.

2. Schaefer GB, Mendelsohn NJ; Professional Practice and Guidelines Committee (2013) Clinical genetics evaluation in identifying the etiology of autism spectrum disorders: 2013 guideline revisions. Genet Med 15: 399-407.

3. Durand CM, Betancur C, Boeckers TM, Bockmann J, Chaste P, et al. (2007) Mutations in the gene encoding the synaptic scaffolding protein SHANK3 are associated with autism spectrum disorders. Nat Genet 39: 25-27.

4. Life technology. Big company provide several brand for laboratory product (DNAzol BD reagent kit and single tube custom TaqMan SNP genotyping assays kit).

5. Abrahams BS, Geschwind DH (2008) Advances in autism genetics: on the threshold of a new neurobiology. Nat Rev Genet 9: 341-355.

6. Lauritsen MB (2013) Autism spectrum disorders. Eur Child Adolesc Psychiatry 22 Suppl 1: S37-42.

7. Bailey A, Palferman S, Heavey L, Le Couteur A (1998) Autism: the phenotype in relatives. J Autism Dev Disord 28: 369-392.

8. Schaefer GB, Mendelsohn NJ (2008) Genetics evaluation for the etiologic diagnosis of autism spectrum disorders. Genet Med 10: 4-12.

9. Müller RA, Pierce K, Ambrose JB, Allen G, Courchesne E (2001) Atypical patterns of cerebral motor activation in autism: a functional magnetic resonance study. Biol Psychiatry 49: 665-676.

10. Johnson NL, Giarelli E, Lewis C, Rice CE (2013) Genomics and autism spectrum disorder. J Nurs Scholarsh 45: 69-78.

11. Hochstenbach R, Buizer-Voskamp JE, Vorstman JAS, Ophoff RA (2011) Genome arrays for the detection of copy number variations in idiopathic mental retardation, idiopathic generalized epilepsy and neuropsychiatric disorders: lessons for diagnostic workflow and research. Cytogenetic and Genome Research, 135: 174-202

12. Shen Y, Dies KA, Holm IA, Bridgemohan C, Sobeih MM, et al. (2010) Clinica genetic testing for patients with autism spectrum disorders. Pediatrics 125: e727-735.

13. Bauer SC, Msall ME (2011) Genetic testing for autism spectrum disorders. Dev Disabil Res Rev 17: 3-8.

14. Horev G, Ellegood J, Lerch JP, Son YE, Muthuswamy L, et al. (2011) Dosagedependent phenotypes in models of 16p11.2 lesions found in autism. Proc Natl Acad Sci U S A 108: 17076-17081.

15. Castro J, Mellios N, Sur M (2013) Mechanisms and therapeutic challenges in autism spectrum disorders: insights from Rett syndrome. Curr Opin Neurol 26 : 154-159.

16. Bourgeron T (2009) A synaptic trek to autism. Curr Opin Neurobiol 19: 231-234 
Citation: Alnefaie G (2014) The Association of Homozygote T Allele of rs2943641 Polymorphism near of Insulin Receptor Substrate 1 Gene in the Susceptibility to Autism. Gene Technology 3: 109. doi: 10.4172/2329-6682.1000109

17. Autism Genome Project Consortium1, Szatmari P, Paterson AD, Zwaigenbaum L, Roberts W, et al. (2007) Mapping autism risk loci using genetic linkage and chromosomal rearrangements. Nat Genet 39: 319-328.

18. Butler MG, Dasouki MJ, Zhou XP, Talebizadeh Z, Brown M, et al. (2005) Subse of individuals with autism spectrum disorders and extreme macrocephaly associated with germline PTEN tumour suppressor gene mutations. Journal of Medical Genetics, 42: 318-321.

19. Bahl S, Chiang C, Beauchamp RL, Neale BM, Daly MJ, et al. (2013) Lack of association of rare functional variants in TSC1/TSC2 genes with autism spectrum disorder. Mol Autism 4: 5.

20. Sun XJ, Rothenberg P, Kahn CR, Backer JM, Araki E, et al. (1991) Structure of the insulin receptor substrate IRS-1 defines a unique signal transduction protein. Nature 352: 73-77.

21. Tzatsos A (2009) Raptor binds the SAIN (Shc and IRS-1 NPXY binding) domain of insulin receptor substrate-1 (IRS-1) and regulates the phosphorylation of IRS-1 at Ser-636/639 by mTOR. J Biol Chem 284: 22525-22534.

22. Ensembl website.

23. Rapin I (1991) Autistic children: diagnosis and clinical features. Pediatrics 87: 751-760.

24. Stamou M, Streifel KM, Goines PE, Lein PJ (2013) Neuronal connectivity as a convergent target of gene $\times$ environment interactions that confer risk for Autism Spectrum Disorders. Neurotoxicol Teratol 36: 3-16.

25. Open SNP website.

26. Frodo Primer3.

27. Laumonnier F, Bonnet-Brilhault F, Gomot M, Blanc R, David A, et al. (2004)
X-linked mental retardation and autism are associated with a mutation in the NLGN4 gene, a member of the neuroligin family. Am J Hum Genet 74: 552-557.

28. Tan GC, Doke TF, Ashburner J, Wood NW, Frackowiak RS (2010) Norma variation in fronto-occipital circuitry and cerebellar structure with an autismassociated polymorphism of CNTNAP2. Neuroimage 53: 1030-1042.

29. Wis'niowiecka-Kowalnik B, Nesteruk M, Peters SU, Xia Z, Cooper ML, et al. (2010) Intragenic rearrangements in NRXN1 in three families with autism spectrum disorder, developmental delay, and speech delay. Am J Med Genet B Neuropsychiatr Genet, 153: 983-993.

30. Blood bank central in Saudi Arabia.

31. Herman GE, Butter E, Enrile B, Pastore M, Prior TW, et al. (2007) Increasing knowledge of PTEN germline mutations: Two additional patients with autism and macrocephaly. Am J Med Genet A 143: 589-593.

32. Luo M, Langlais P, Yi Z, Lefort N, De Filippis EA, et al. (2007) Phosphorylation of human insulin receptor substrate-1 at Serine 629 plays a positive role in insulin signaling. Endocrinology 148: 4895-4905.

33. Maglio C, Ericson U, Burza MA, Mancina RM, Pirazzi C, et al. (2013) The IRS1 rs2943641 variant and risk of future cancer among morbidly obese individuals J Clin Endocrinol Metab 98: E785-789.

34. Prince Salman Central for Disability Research in Saudi Arabia.

35. Rasmussen P, Börjesson O, Wentz E, Gillberg C (2001) Autistic disorders in Down syndrome: background factors and clinical correlates. Dev Med Child Neurol 43: 750-754.

36. Rung J, Cauchi S, Albrechtsen A, Shen L, Rocheleau G, et al. (2009) Genetic variant near IRS1 is associated with type 2 diabetes, insulin resistance and hyperinsulinemia. Nat Genet 41: 1110-1115 